\title{
Personalized Psychiatry and Depression: The Role of Sociodemographic and Clinical Variables
}

\author{
Giampaolo Perna ${ }^{1,2,3,4}$, Alessandra Alciati ${ }^{2,5}$, Silvia Daccò ${ }^{1,2}$, Massimiliano Grassi ${ }^{1,2}$, and Daniela Caldirola ${ }^{1,2}$ \\ ${ }^{1}$ Humanitas University Department of Biomedical Sciences, Milan, Italy \\ ${ }^{2}$ Department of Clinical Neurosciences, Villa San Benedetto Menni Hospital, Hermanas Hospitalarias, Como, Italy \\ ${ }^{3}$ Department of Psychiatry and Neuropsychology, Faculty of Health, Medicine and Life Sciences, Maastricht University, Maastricht, The Netherlands \\ ${ }^{4}$ Department of Psychiatry and Behavioral Sciences, Leonard Miller School of Medicine, Miami University, Miami, USA \\ ${ }^{5}$ Humanitas Clinical and Research Center, IRCCS, Milan, Italy
}

\begin{abstract}
Despite several pharmacological options, the clinical outcomes of major depressive disorder (MDD) are often unsatisfactory. Personalized psychiatry attempts to tailor therapeutic interventions according to each patient's unique profile and characteristics. This approach can be a crucial strategy in improving pharmacological outcomes in MDD and overcoming trial-and-error treatment choices. In this narrative review, we evaluate whether sociodemographic (i.e., gender, age, race/ethnicity, and socioeconomic status) and clinical [i.e., body mass index (BMI), severity of depressive symptoms, and symptom profiles] variables that are easily assessable in clinical practice may help clinicians to optimize the selection of antidepressant treatment for each patient with MDD at the early stages of the disorder. We found that several variables were associated with poorer outcomes for all antidepressants. However, only preliminary associations were found between some clinical variables (i.e., BMI, anhedonia, and MDD with melancholic/atypical features) and possible benefits with some specific antidepressants. Finally, in clinical practice, the assessment of sociodemographic and clinical variables considered in our review can be valuable for early identification of depressed individuals at high risk for poor responses to antidepressants, but there are not enough data on which to ground any reliable selection of specific antidepressant class or compounds. Recent advances in computational resources, such as machine learning techniques, which are able to integrate multiple potential predictors, such as individual/ clinical variables, biomarkers, and genetic factors, may offer future reliable tools to guide personalized antidepressant choice for each patient with MDD.

Psychiatry Investig 2020;17(3):193-206
\end{abstract}

Key Words Depression, Personalized medicine, Drug targeting, Clinical markers, Antidepressants, Treatment outcome.

\section{INTRODUCTION}

The World Health Organization estimates that depression will be the second-most common cause of disease and premature death worldwide by $2020 .{ }^{1}$ Moreover, depression is expected to be the largest contributor to disease burden by $2030 .^{2}$ Huge personal and societal costs are associated with the disability caused by major depressive disorder (MDD), which frequently arises from the poor response to the current therapeutic options. ${ }^{3}$

Received: October 28, 2019 Revised: December 9, 2019

Accepted: January 14, 2020

$\triangle$ Correspondence: Giampaolo Perna, MD, PhD

Humanitas University Department of Biomedical Sciences, Via Rita Levi Montalcini 4, 20090 Pieve, Emanuele-Milan, Italy

Tel: +39 028224 7100, E-mail: giampaolo.perna@hunimed.eu

(c) This is an Open Access article distributed under the terms of the Creative Commons Attribution Non-Commercial License (https://creativecommons.org/licenses/by$\mathrm{nc} / 4.0$ ) which permits unrestricted non-commercial use, distribution, and reproduction in any medium, provided the original work is properly cited.
Recent guidelines on pharmacological treatments of MDD indicate the use of selective serotonin reuptake inhibitors (SSRIs) or serotonin noradrenaline reuptake inhibitors (SNRIs) as first-line treatment, along with other antidepressants (ADs), including agomelatine, bupropion, mirtazapine, and vortioxetine. Tricyclic antidepressants (TCAs), trazodone, levomilnacipran, and vilazodone are recommended as second-line agents, whereas third-line recommendations include monoamine oxidase inhibitors (MAOIs) and the SNRI, reboxetine. ${ }^{4}$ Although concerns of the U.S. Food and Drug Administration (FDA) about antidepressant-associated risk of suicidality in young adults are still a matter of debate, most clinicians and researchers consider that antidepressant-associated benefits overweigh risks in individuals with depression. ${ }^{5}$ Despite these pharmacological options, the clinical outcomes are often unsatisfactory. The response to first-line treatment is estimated to be between $40 \%$ and $60 \%,{ }^{6-8}$ while only $30-53 \%$ of patients achieve a full remission after antidepressant treat- 
ment. ${ }^{6-8}$ Furthermore, approximately $35 \%$ of the patients with MDD who fail to respond to first-line treatment obtain a remission after switching to a second-line treatment., ${ }^{9,10}$ Finally, between $34 \%$ and $48 \%$ of depressed patients fail to respond to two or more adequate courses of AD medications. ${ }^{11,12}$

A possible reason for the high rate of unsatisfactory responses to ADs is that MDD is a very heterogeneous disorder with respect to symptom presentation and, possibly, its underlying mechanisms. ${ }^{13}$ According to the Diagnostic and Statistical Manual of Mental Disorders-5th ed. (DSM-5) diagnostic criteria, ${ }^{14}$ there are more than 60 forms of MDD, given the various possible combinations of symptoms by which a major depressive episode (MDE) can be diagnosed. ${ }^{15}$ The symptoms include depressed mood and/or a loss of interest and pleasure (anhedonia), and at least four other symptoms among a list of seven. Furthermore, additional symptomatological features can be considered as specifiers of each MDE. ${ }^{14}$

In this scenario, it is plausible that subgroups of depressed individuals, possibly sharing similar clinical and pathophysiological characteristics, are better suited to some medications, whereas others may obtain limited benefits from the same treatments. Personalized psychiatry can be a crucial strategy to improve pharmacological responses in MDD. This approach attempts to tailor therapeutic interventions according to each patient's unique profile and characteristics, by integrating information from clinical features, biomarkers, genetic/epigenetic factors, and environmental influences, with the final aim of optimizing the choice among treatment options when facing a current MDE, thus overcoming trial-anderror treatment choices. As the lack of full remission of an MDE is associated with high recurrence of episodes, chronic course, and more severe functional impairment, ${ }^{16,17}$ increasing the chances of successful therapeutic responses during an MDE can play a key role in making the global course and outcomes of MDD more favorable.

In clinical practice, clinicians already use a somewhat personalized strategy, combining personal experience and scientific evidence, to choose a tailored treatment for each patient. However, personal beliefs and interpretative models, not sufficiently grounded on scientific evidence, may lead to bias in treatment selection. Therefore, efforts to give some evidencebased suggestions in the framework of personalized medicine could have positive effects on clinicians' decisions.

In this narrative review, we evaluated if, and to what extent, some variables easily assessable in clinical practice through clinical interviews may contribute to optimizing the pharmacological choice for each patient with an MDE at the early stage of the disorder. Given the extensiveness of the topic, we focused only on antidepressant medications, while we did not consider combined medications, psychological interventions, or treatments targeted on non-responder/treatment-resistant patients. Specifically, we considered the influence of some sociodemographic and clinical [i.e., body mass index (BMI), and severity and profile of depressive symptoms] variables on responses to antidepressant classes and/or specific antidepressant compounds.

If we find significant results, it may increase the possibility that a personalized medicine approach becomes part of everyday clinical practice in psychiatric settings when more sophisticated, time-consuming, and expensive sources of predictions are unavailable.

\section{SOCIODEMOGRAPHIC VARIABLES}

\section{Gender}

A meta-analysis of 35 historical trials conducted between 1957 and 1991 suggested that men benefited more from the TCA imipramine than premenopausal women. ${ }^{18}$ Similarly, a subsequent study in a large sample showed that premenopausal women responded significantly faster and better to the SSRI sertraline than to imipramine, whereas the opposite was found in men, and postmenopausal women had similar rates of response to the two medications. ${ }^{19}$ A later review of 15 randomized, placebo-controlled trials (RCTs) supported the possibility of better responses to SSRIs in women when compared to men. ${ }^{20}$ Consistently, a meta-analysis suggested a possible better response of females to the SSRI paroxetine, ${ }^{21}$ while a metaregression analysis of 59 studies found a possible better trend in females after 6 weeks-treatment with sertraline..$^{22}$ In line with these findings, preliminary results in a small sample of young men and premenopausal women suggested a greater response in females treated with the SSRI citalopram than in females treated with the SNRI reboxetine, while no differences were observed in men. ${ }^{23}$ Moreover, the analysis of a pooled dataset from eight RCTs found among women (not men) showed better responses to SSRIs in younger patients ( $<50$ years); hormone replacement therapy appeared to eliminate this difference among women. ${ }^{24}$ Higher remission rates in females were found in the large-scale trial Sequenced Treatment Alternatives to Relieve Depression (STAR*D) (Phase 1) after 14 weeks of treatment with citalopram in psychiatric and primary care "real world" settings. ${ }^{25}$ Finally, some indications of more favorable responses to MAOIs ${ }^{26}$ and the SNRI venlafaxine in females were found. ${ }^{20,21}$ However, many authors claim that these gender differences in treatment response, although statistically significant, are not large enough to suggest that gender should guide the clinical use of antidepressants. ${ }^{20,21,26,27}$ Moreover, other findings failed to reveal any gender effects in predicting or moderating differential responses to different antidepressant classes or compounds. Indeed, retrospective 
examinations of multiple RCTs did not find gender differences in responses to TCAs, the SSRI fluoxetine, ${ }^{26,28}$ or venlafaxine; ${ }^{24}$ similarly, no effects of gender or menopausal status on treatment outcomes with venlafaxine or fluoxetine were found in a large, multiphase, multicenter trial. ${ }^{29}$ Likewise, a metaanalysis of 30 RCTs found that responses to the TCAs imipramine and amitriptyline were independent of gender, even when considering interactions between gender and age. ${ }^{30} \mathrm{~A}$ more recent meta-analysis of multiple RCTs further supports the lack of associations between gender and responses to TCAs (imipramine, nortriptyline) or SSRIs (paroxetine, escitalopram, fluoxetine).$^{31}$ Finally, in the International Study to Predict Optimized Treatment in Depression (iSPOT-D), no gender differences were found after 8 weeks of randomized treatment with escitalopram, sertraline, or venlafaxine. ${ }^{32}$

In conclusion, many studies did not find gender effects on clinical response to antidepressants in MDD and, even when statistically significant differences between females and males were found, they appeared to be small and probably not relevant from a clinical point of view. Therefore, so far, gender does not seem to be a reliable factor to consider when choosing an antidepressant class or specific compound for MDD in clinical practice.

\section{Age}

Studies investigating the relationships between age and response to antidepressants in MDD have yielded inconsistent results. A recent review including RCTs, uncontrolled treatment trials, and observational studies, suggests that older current age and earlier age at onset of MDD may have direct effects on poor overall treatment response. ${ }^{33}$ These findings were partly supported by a recent individual participant data (IPD) meta-analysis of Japanese RCTs, comparing bupropion, duloxetine, escitalopram, paroxetine, mirtazapine, and venlafaxine with placebo in the first 6-8 weeks of treatment. ${ }^{34} \mathrm{In}$ deed, in line with the review previously mentioned, ${ }^{33}$ the IPD meta-analysis indicated that the older the current age, the smaller the difference in depression severity reduction between antidepressants and placebo, while, in the opposite direction, it found that the older the age at onset the smaller the superiority of the antidepressants over placebo. ${ }^{34}$ Finally, recent findings from the 8-week multisite EMBARC trial of sertraline versus placebo found that older current age was associated with better outcomes to sertraline than placebo. ${ }^{35}$ Conversely, both the STAR*D (Phase 1$)^{25}$ and the iSPOT- ${ }^{32}$ studies failed to find relationships between current age or age at onset and response to antidepressant treatments.

In conclusion, the mixed available results do not allow clinicians to consider current age or age at onset as reliable variables to guide the selection of an antidepressant treatment for MDD in clinical practice.

\section{Race and ethnicity}

Older studies, performed with very different methodology concerning design, settings, outcome measures, and types/ doses of medications, provided inconsistent results about the possible influence of ethnic differences on antidepressant treatment outcomes in MDD. Some findings suggested that African-Americans and Latinos may respond to lower doses of antidepressants when compared with Caucasian patients, ${ }^{36,37}$ whereas others found poorer global outcomes for minority patients than Caucasians. ${ }^{38,39}$ More recently, pooled analyses of large pharmacy-sponsored databases found similar response and remission rates to paroxetine and duloxetine treatments in Latino, African American, and Asian American patients compared with whites, ${ }^{40,41}$ even though these findings may not be fully representative of treatment effectiveness in clinical settings. Partly in line with these findings, the "real world" clinical study STAR*D (Phase 1 ) found only few indications in blacks, and no indications in Hispanics, of poorer outcomes to citalopram treatment compared with whites, after adjustment for many baseline clinical, demographic, and socioeconomic differences. ${ }^{42}$ Specifically, remission rate remained worse for blacks in only one of several outcome measures, i.e., the self-report version of the 16-item Quick Inventory of Depressive Symptomatology. ${ }^{42}$ A prospective 8-week, open-label clinical trial with citalopram found similar outcomes in African-American and Caucasian patients with MDD, despite several baseline differences in demographic/ socioeconomic variables and depression severity. ${ }^{43}$ Similar findings were obtained in the Combining Medications to Enhance Depression Outcomes (CO-MED) trial, which examined if outcomes differed by race-ethnicity (i.e., non-Hispanic whites, blacks, and white Hispanics) in the acute (12 weeks) and continuation (12-28 weeks) phases of randomized treatment with escitalopram (plus placebo) or one of two antidepressant combinations (bupropion plus escitalopram/venlafaxine plus mirtazapine). Although black participants had greater baseline psychiatric and medical comorbidities, there were no significant differences in clinical outcomes between groups. ${ }^{44}$ Likewise, the iSPOT-D study did not find associations between response to antidepressant treatment and race. ${ }^{32}$ Taken together, the results of these studies suggest that socioeconomic disparities were likely contributors to the ethnic differences in treatment outcomes found in older studies; hence most differences may be overcome by improving access to and quality of care for minority groups. ${ }^{44}$ However, it should be noted that subsequent re-analyses of STAR*D data to examine independent contributions of race and genetic ancestry to citalopram response found that, although socio- 
economic and baseline clinical factors drove racial differences in antidepressant response, genetic African ancestry, rather than self-reported race, explained a significant fraction of the residual differences between blacks and whites. ${ }^{45}$

In conclusion, no specific indications based on race/ethnicity are available when choosing an antidepressant treatment in clinical practice. However, the possible influence of low socioeconomic status and genetic African ancestry on poorer outcomes, as well as some indications of higher attrition rates of blacks in both clinical trials and clinical sites of care, ${ }^{44,46}$ should be considered in clinical practice when selecting optimal setting and intensity of care for ethnic minority groups.

\section{Socioeconomic variables}

A large body of literature supports the association between low socioeconomic status (SES) and both higher prevalence of depression and higher risk of developing it over time..$^{47,48}$ Several studies suggest that SES also influences the outcomes of antidepressant treatments. In the STAR*D study (Phase 1), socioeconomic factors, such as low income, education, and unemployment, were highly discriminative in predicting a poor response to citalopram, even with disparities in access to care accounted for, ${ }^{25,49}$ whereas reviews of several studies found that high income and SES, as well as living with a spouse/partner, were predictive of a better response to antidepressants. ${ }^{50,51}$ If unemployment is consistently considered a risk factor of poorer antidepressant response, recent findings suggest than even the occupational level of patients in employment may play a role in treatment outcome. In a large multicenter and multinational study project [Group for the Study of Resistant Depression (GSRD)], patients with high occupational level (OL) (e.g., higher executives/business managers/administrative personnel) showed lower response/remission with the last treatment (SSRIs, SNRIs, and NRIs) for the current depressive episode, compared with those with middle or low occupational level. Moreover, the high OL group contained a higher proportion of patients who suffered from at least two consecutive failures of treatment. ${ }^{52} \mathrm{~A}$ following study, with an independent sample, confirmed that managers and white-collar workers had higher rates of non-response and resistance to antidepressant treatments than blue-collar workers. ${ }^{53}$ These unexpected results may be partly explained by some specific stressful psychosocial factors related to high OL that may hinder the healing process, such as high demand and workload, pressure, competition, social isolation at work, work-life imbalance, and personal attributes leading to difficulties in adapting to the depressive state or stigma of mental illness. ${ }^{53}$

In conclusion, the available findings suggest that assessment of SES and OL in clinical practice can help to select patients that need special care over the course of treatment for increased risk of poor responses to antidepressants, whereas no indications are available to guide a personalized class- or medication-specific selection.

\section{CLINICAL VARIABLES}

\section{Body mass index}

Obesity is one of the most prevalent comorbidities of MDD, in particular among women, ${ }^{54}$ and it is associated with more severe and chronic MDD. ${ }^{55}$ This relationship raises the question if being overweight or obese may be associated with a poor response to antidepressants, and if BMI may inform the selection of antidepressant treatment.

In an older meta-analysis of three clinical trials (all doubleblind, active-controlled comparisons of marketed SSRIs and SNRIs, conducted between 2003 and 2006), being overweight (i.e., BMI between 25.0 and 29.9) and, to a greater extent, obese (i.e., $\mathrm{BMI} \geq 30$ ) were predictive of poor outcomes compared to normal weight. ${ }^{56}$ Subsequent studies confirmed the association between higher weight and poorer responses to antidepressants in MDD, while the findings regarding specific classes or compounds were heterogeneous. A pooled analysis of Phase II-IV SSRIs-clinical trials, involving obese/non-obese participants, showed that the subgroup of depressed obese men had little or no therapeutic benefit with SSRIs compared with the rest of the depressed sample. ${ }^{57}$ Similarly, increased BMI was associated with poorer outcomes in two short-term trials with the SSRI fluoxetine. ${ }^{58,59}$ Conversely, overweight and obesity did not seem to influence responses to the SSRI escitalopram in a very large sample participating in the Genome Based Therapeutic Drugs for Depression (GENDEP) project (a 12-week open-label, part-randomized, multicenter trial comparing treatment with escitalopram or nortriptyline), ${ }^{60}$ while in the same sample, these features predicted poor responses to the TCA nortriptyline..$^{60}$ More recently, two reviews supported the association between obesity/overweight and poor antidepressant responses, even though it was difficult to draw definitive conclusions due to the methodological differences among studies. ${ }^{61,62}$ Subsequent findings from the iSPOT-D trial revealed that venlafaxine monotherapy was more effective than escitalopram in obese II (BMI between 35.0 and -39.9$)$ and obese III (BMI $\geq 40)$ depressed outpatients, while escitalopram was more effective than venlafaxine in those with normal BMI. ${ }^{63}$

A recent secondary analysis of data from the CO-MED trial found that normal- or under-weight participants (BMI $<25$ ) appeared to be less likely to remit with the bupropionescitalopram combination than with escitalopram monotherapy or the venlafaxine-mirtazapine combination, whereas 
obese II+ participants (BMI $\geq 35)$ were more likely to remit with bupropion-escitalopram than the other two treatment options. ${ }^{64}$ These results support and extend those of Green and colleagues. ${ }^{63}$ Indeed, the dopaminergic and noradrenergic antidepressant bupropion in combination with escitalopram was supposed to have a pharmacological profile similar to that of venlafaxine, which provided greater benefits than escitalopram in iSPOT-D. ${ }^{64}$ Taken together, these studies provide very preliminary suggestions of personalized, medicationspecific, antidepressant selection in clinical practice based on BMI measurements. Bupropion-escitalopram combination should be avoided in normal-/under-weight depressed outpatients, as compared to escitalopram monotherapy or venlafaxine-mirtazapine combination, while it should be preferred in those with $\mathrm{BMI} \geq 35$. The latter group may also benefit from venlafaxine monotherapy, which, conversely, may be less efficacious in normal-/under-weight depressed patients, as compared to escitalopram monotherapy. Finally, fluoxetine and nortriptyline may be less efficacious in overweight/obese depressed patients.

In conclusion, most findings point to associations between overweight/obesity and poorer responses to antidepressants, which suggest careful monitoring of symptoms during the course of treatment in depressed patients with these features. Only preliminary indications are available concerning antidepressant-specific selection based on BMI.

\section{Severity of depressive symptoms}

One of the most studied clinical predictors of antidepressant treatment outcome is pretreatment depressive symptom severity. A meta-analysis by Khan and colleagues ${ }^{65}$ found that greater baseline depression severity was associated with significantly greater magnitude of symptom reduction during antidepressant treatment compared to placebo. In line with these results, a subsequent meta-analysis ${ }^{66}$ concluded that lower depressive symptom severity predicted minimal to no advantage of antidepressants over placebo. Conversely, the magnitude of the advantage in taking antidepressants increased as the severity of pretreatment depression increased, ${ }^{35,66}$ although the very severely depressed patients had lower probabilities of achieving a full remission of depressive symptoms than a clinical response (i.e., symptom reduction), and displayed less favorable global outcomes. ${ }^{51,67}$ In line with these findings, recent results from the European GSRD indicated that great depressive symptom severity, as well as early age of onset ( $\leq 18$ years), are predictors of resistance to $\mathrm{AD}$ treatment. ${ }^{68}$

In conclusion, the greater the pretreatment symptom severity of patients in an MDE, the greater the expected treatment response to the whole class of antidepressants, even if, in patients with severe depressive symptoms, full remission seemed to be more difficult to achieve than response and poorer treatment outcomes are more likely. Patients with very low depressive symptom severity may not require antidepressants, while non-pharmacological interventions, such as cognitive behavioral therapy (CBT), may be sufficient for symptom remission. ${ }^{69}$ No indications were available for personalized selection of specific antidepressant class or compound based on the range of depressive severity.

\section{Symptom profiles}

Symptom profile-based predictors and moderators of treatment response were widely investigated in research studies, as the examination of symptomatological expression of an MDE is usually part of the psychiatric interview. Symptom profiles include single specific depressive symptoms, such as anhedonia, symptom dimensions (i.e., groups of co-occurring symptoms identified through factor analyses of depression rating scales), subtypes, and specifiers. However, while the definition of single depressive symptoms is more univocal, considerable variations were found in the literature for the definition of depressive subtypes/specifiers, with risk of subjective and idiosyncratic interpretations. As an example, although both the DSM-IV-TR and DSM-5 made a distinction between subtypes (i.e., mutually exclusive and jointly exhaustive phenomenological subgroupings) and specifiers (i.e., non-mutually exclusive or jointly exhaustive phenomenological subgroupings), ${ }^{14,70}$ these two terms are often used interchangeably in scientific studies. Furthermore, both the criteria used to define the same construct, such as melancholic depression, and methods of assessment (e.g., clinical interview/psychometric tools; self-administered/clinician-administered scales) vary considerably across studies, thus making the results not always directly comparable. Therefore, the available findings should be interpreted keeping in mind the limitations affecting this body of research.

Given the extensiveness of the topic, we specifically focused on anhedonia, a core symptom of major depression, and MDD with melancholic features, with atypical features and with anxiety symptoms.

\section{Anhedonia}

Anhedonia (i.e., the loss of pleasure in activities, and/or lack of reactivity to usually pleasurable stimuli) is considered a cardinal symptom of MDD, and it was proposed as a possible endophenotype of depression. ${ }^{71}$ Anhedonia is thought to reflect the dysfunction of the brain reward circuits, which are crucial for both the motivational drive and the ability to feel pleasure in response to events/activities (i.e., consummatory pleasure) ${ }^{71-73}$ Findings from preclinical and clinical studies suggest the centrality of dopamine in motivational aspects of 
reward processing, while endogenous opioids may be involved in the hedonic experience. ${ }^{7475}$ More recently, other neurotransmitters, such as glutamate, have broadened the picture of mediation of reward and anhedonia at neurotransmitter level. ${ }^{73,76}$

From a clinical point of view, some studies point to relationships between anhedonia and severe behavioral and somatic MDD-related events. ${ }^{58,77,78}$ For these reasons, it is of great importance when treating MDD to consider the therapeutic effectiveness of medications on this critical aspect of the disorder. Unfortunately, at present, there are no approved medications specifically targeting anhedonia, which is associated with poorer responses to treatments and poorer short-/ long-term outcomes. ${ }^{79,80}$ In the GENDEP and STAR*D (Phase 1) studies, higher interest-activity symptom dimension at baseline, mainly reflecting anhedonia, was the most robust predictor of a poor response to escitalopram or nortriptyline (GENDEP), and to citalopram (STAR*D), irrespective of overall depression severity and multiple sociodemographic variables.$^{81}$ Likewise, other studies found that the current standard antidepressant treatments, particularly SSRIs, did not seem to be fully effective in treating reward-related symptoms of depression, ${ }^{82,83}$ and anhedonia often seemed to persist despite the resolution of other symptoms of depression. ${ }^{84}$ Moreover, several studies suggested that SSRIs may induce some clinical features close to anhedonia in depressed patients, such as emotional blunting and restriction in the range of emotions ${ }^{85}$ possibly related to SSRI induced dampening of dopaminergic/noradrenergic activity. ${ }^{86}$ This possibility may also partly explain why anhedonia and fatigue often remained in some depressed patients with an overall positive response to SSRIs. ${ }^{87}$

In this context, a recent systematic review ${ }^{88}$ examined the $^{8}$ issue whether specific antidepressants may be particularly suitable to treat anhedonia and provided some preliminary results from relatively few comparative pharmacological studies that used reliable measures of anhedonia. The antidepressant agomelatine, which has melatonergic activity, increases serotonin and dopamine levels in the frontal cortex, and enhances expression of brain-derived neurotrophic factor (BDNF), ${ }^{89,90}$ reduced emotional blunting and affective flattening in some open-label studies, ${ }^{88}$ and it displayed greater efficacy on measures of anhedonia when compared with the SNRI venlafaxine extended-release (ER), ${ }^{91}$ or the SSRI escitalopram. ${ }^{92}$ Conversely, no significant differences in antianhedonic properties were found in a randomized double-blind trial comparing the SSRI fluoxetine with ER venlafaxine. ${ }^{93}$ The reversible MAOIA moclobemide, which has pro-dopaminergic properties, had earlier and greater antianhedonic effects when compared with the serotonergic TCA clomipramine in a 4-week double-blind multicenter study ${ }^{94}$ Similarly, the norepinephrine-dopamine reuptake inhibitor, bupropion, appears to alleviate anhedonia by improving reward response, interest, and energy when compared with placebo. ${ }^{95}$ Recent findings of an open-label trial ${ }^{96}$ suggest that a single infusion of ketamine $(0.5 \mathrm{mg} / \mathrm{kg})$, a medication targeting the glutamatergic system through its NMDA receptor antagonistic properties, may provide rapid (i.e., during the first three days post-infusion) antianhedonic effects, which was maintained for up to 28 days post-infusion. Overall, there was significant methodological heterogeneity across the studies included in the qualitative review of Cao and colleagues, ${ }^{88}$ such as trial duration, illness severity, medication dose, and assessment measure. Additionally, the number of available studies was limited. For these reasons, the results should be considered preliminary, and more research is needed to provide any ranking of the antianhedonic properties of different antidepressants.

In conclusion, the association between anhedonia and poorer responses to antidepressants suggest to clinicians a careful monitoring of patients with this prominent symptom during the course of antidepressant treatment to select the intensity of care and consider complementary strategies, such as behavioral activation, physical exercise, and/or pharmacotherapeutic combination or augmentation strategies. ${ }^{81}$ Only very preliminary indications are available for clinicians concerning possible antidepressant-specific selection based on the presence of anhedonia.

\section{MDD with melancholic features}

According to the DSM-IV-TR and DSM-5, ${ }^{14,70}$ MDD with melancholic features (MDD-MF) is characterized by pervasive anhedonia, accompanied by at least three other symptoms among a distinct quality of depressed mood (e.g., profound despondency/moroseness), worse depression in the morning, early morning awakening, psychomotor agitation/retardation, significant anorexia/weight loss, and inappropriate guilt.

Studies comparing efficacy of antidepressants between melancholic and non-melancholic depression provided controversial results concerning both the outcome to antidepressants considered as a group and to different types of antidepressants. These discrepancies were thought to be related to methodological differences across studies concerning design, definition/assessment of melancholia, differences in baseline features of patients, and statistical power.

In both the STAR*D (Phase 1) ${ }^{97}$ and iSPOT-D ${ }^{98}$ studies, unadjusted analyses found a lower rate of remission in patients with MDD-MF than those without melancholic features, but differences became non-significant after adjusting for baseline characteristics. Furthermore, no indications were found that the melancholic/non-melancholic features may guide antidepressant selection. ${ }^{99}$ Similarly, secondary analyses of data 
from the CO-MED trial found no evidence of differential remission or response rates to antidepressant combination or monotherapy between melancholic/non-melancholic MDD patients. ${ }^{100}$ Conversely, in a prospective, naturalistic, multicenter, nationwide epidemiological Spanish study, ${ }^{101}$ outpatients with MDD-MF, compared with non-melancholic outpatients, showed lower rates of remission after both 6-8 and 14-20 weeks of different antidepressant treatments (SSRIs, SNRIs, and TCAs), even after considering baseline differences between the two groups. A secondary analysis of data from the GENDEP study found that MDD-MF had slightly worse outcomes than non-melancholic MDD, which was relatively specific to escitalopram, but not to nortriptyline. However, the melancholia-drug interaction was not statistically significant on the primary outcome measure, and significant results for secondary measures were not confirmed in sensitivity analyses restricted to randomly allocated individuals; hence the authors concluded that MDD-MF was not a sufficiently robust differential predictor of outcome to guide clinicians in choosing between SSRIs and TCAs. ${ }^{102}$ In the opposite direction, in a nationwide Korean naturalistic study, patients with MDD-MF who received 12-week, clinician-determined, antidepressant intervention had faster and higher rates of remission, especially when SSRIs were used, than those without melancholic features, even after adjustment for baseline status. $^{103}$

The group of studies that specifically focused on the clinical response to different types of antidepressants provided inconsistent results. Briefly, older studies initially discouraged the use of MAOIs in MDD-MF, while subsequent results did not find differences between MAOIs and TCAs. First studies with SSRIs suggested lower effectiveness of these compounds in MDD-MF compared with TCAs, while more recent studies found heterogeneous results ranging from better outcomes with TCAs to no differences, or to better response with SSRIs. Finally, inconsistent results were also found in comparisons between SSRIs and newer antidepressants such as venlafaxine. For a detailed list of these studies, please refer to the review cited below. ${ }^{104}$

Recently, a comprehensive meta-analysis attempted to clarify this heterogeneity. It included randomized and nonrandomized trials that were published between 1980 and 2017 and using validated measures to define melancholic/nonmelancholic MDD. ${ }^{104}$ Considering antidepressants as a group, MDD-MF, compared with non-melancholic MDD, was less likely to show response to placebo (weighted mean follow-up period, 5.8 weeks) and was significantly associated with decreased likelihood of remitting from the MDE (weighted mean follow-up period, 10.6 weeks), even taking into account the higher baseline severity of depressive symptomatology found in MDD-MF. Conversely, MDD-MF was not associated with a decreased likelihood of responding to antidepressants during a weighted mean follow-up period of 10 weeks. A possible explanation of this discrepancy is that MDD-MF may take a longer time to remit than non-melancholic MDD, as found in a previous study, ${ }^{105}$ while the mean duration of trials was sufficient for a $50 \%$ reduction in baseline depressive symptoms (i.e., "response"), thus making the odds of response in melancholic/non-melancholic MDD similar. ${ }^{104}$ Comparing the odds of remission to different types of antidepressants in MDDMF, melancholic patients treated with SSRIs had significantly lower odds of achieving remission than those treated with TCAs, while no differences were found between SSRIs and venlafaxine. $^{104}$

In conclusion, considering the relative shortage of studies specifically focusing on MDD-MF, and the methodological limitations of this body of research, the available findings should be considered preliminary and further research is needed. ${ }^{104}$ Due to the central role of anhedonia in MDD-MF, future studies should also evaluate the efficacy of compounds that seem promising when tested on anhedonia considered as a single symptom, as described in the previous section. So, far, provisional conclusions suggest that MDD-MF may have lower rate of remission than non-melancholic MDD with antidepressant treatments, and TCAs may have higher efficacy than SSRIs in patients with MDD-MF. However, due to the well-known less favorable side effect profile of TCAs compared with SSRIs, clinicians should carefully consider the advantages and disadvantages of using TCAs for each individual patient with MDD-MF.

\section{MDD with atypical features}

MDD with atypical features (MDD-AF) describes a condition with significant mood reactivity in response to positive events, and at least two of the following symptoms: interpersonal rejection sensitivity, increased appetite/weight, hypersomnia, and leaden paralysis. ${ }^{14,70}$ Similar to MDD-MF, a mixed picture has arisen for outcomes for antidepressants in MDD-AF, especially concerning the value of atypical features for antidepressant selection. Regarding the global outcome for antidepressants, although some results suggest lower remission rates in MDD-AF than in MDD without atypical features, ${ }^{101}$ most other studies failed to find outcome differences after adjustment for pretreatment baseline differences, including large trials such as iSPOT-D, STAR*D, and GENDEP. ${ }^{99,102,106}$ Concerning preferential response to specific antidepressants, older studies suggest a poorer response to the TCA imipramine in MDD-AF, ${ }^{107}$ and higher efficacy of the irreversible MAOI phenelzine and the reversible MAOI moclobemide compared with imipramine ${ }^{108}$ or the SSRI fluox- 
etine, ${ }^{109}$ respectively. However, another study did not find outcome differences between moclobemide and the SSRI sertraline in MDD-AF. ${ }^{110} \mathrm{~A}$ meta-analysis of double-blind, 6- to 12-week controlled studies, covering the years from 1966 through 2004, supported the higher efficacy of phenelzine and moclobemide over imipramine in MDD-AF, while it provided preliminary suggestions, based on a limited number of studies, that fluoxetine and sertraline may have similar efficacy to MAOIs. ${ }^{111}$ More recently, a 6-week, randomized, double-blind trial found that moclobemide was superior to the TCA clomipramine in MDD-AF, thus supporting the higher efficacy of MAOIs over TCAs in patients with atypical features. ${ }^{112}$ However, as the current use of MAIOs in clinical practice is limited, some subsequent studies focused on SSRIs or other antidepressants newer than MAOIs. Unfortunately, this body of research is still limited. In the GENDEP study, the SSRI escitalopram and the TCA nortriptyline were equally effective in treating MDD-AF, ${ }^{102}$ and no differences were found in the iSPOT-D study when the effectiveness of escitalopram, sertraline, and venlafaxine in MDD-AF were compared. ${ }^{99}$

In conclusion, beyond the older findings of the superiority of MAOIs compared to TCAs in treating MDD-AF, so far, no specific indications are available to help clinicians in selection among newer antidepressants. ${ }^{113}$ However, as associations between atypical depression and bipolarity were found, which can have therapeutic implications, ${ }^{114}$ careful information about possible risk factors for bipolarity should be collected in clinical practice before starting treatment when atypical features are present. Likewise, some associations found between atypical depression, overweight/obesity, and resistance to leptine $\mathrm{e}^{114,115}$ suggest that clinicians should consider antidepressants with limited effects on appetite and/body weight when treating patients with MDD-AF.

\section{MDD with anxiety symptoms}

To take into account the presence of significant anxiety condition in MDD, the DSM-5 introduced the anxious distress specifier, defined as the occurrence, during most days of an MDE, of at least two of five anxiety symptoms. ${ }^{14}$ Before the development of this relatively new DSM- 5 specifier, the concurrence of anxiety symptoms in depression was generally denominated as "anxious depression." This term was used interchangeably across studies to indicate heterogeneous definitions that had poor agreement ${ }^{116}$ and made it more difficult to compare the results. The most common definitions of "anxious depression" included MDD with comorbid full-blown anxiety disorders (AnxDs), or MDD with clinically significant anxiety symptoms measured on different anxiety dimensional rating scales. For the dimensional definition of "anxious depression," the most common criteria were a cut-off score of at least seven on the Hamilton Depression Rating Scale (HAMD) anxiety/somatization factor (ASF), or varying cut-off scores on the Hamilton Anxiety Rating Scale (HAM-A). ${ }^{117}$ Despite heterogeneity in definitions and assessment tools, "anxious depression" was reported in $42-78 \%$ of patients with MDD, and it was generally associated with greater depression chronicity and severity, higher suicide ideation/risks, greater functional disability, lower quality of life, and poorer outcomes compared with MDD without anxiety. ${ }^{117}$ Recently, the DSM5 anxious distress specifier appeared to outperform the presence of comorbid DSM-IV-AnxDs as a longitudinal predictor of depression outcome, suggesting that it may represent a general marker of anxiety able to capture a specific construct with clinical validity and not fully overlap with the co-occurrence of AnxDs in MDD. ${ }^{118}$ In the following paragraphs, we specifically focus on MDD with significant anxiety symptoms (MDD-AS), as identified by both the DSM- 5 specifier and previous dimensional measurements, because the therapeutic implications of comorbidity of MDD with AnxDs require a dedicated review.

Most studies suggest that MDD-AS has poorer outcomes with antidepressants compared to MDD without anxiety symptoms. A review of pharmacological studies (with different designs/compounds), covering the years from 1949 through the beginning of 2013, found that patients with MDD-AS, mostly defined as HAM-D-AFS score $\geq 7$, did not maintain sustained response or remission following initial successful treatments with SSRIs, SNRIs, or TCAs, and, moreover, they were at greater risk of side effects. ${ }^{119}$ In the Phase 1, STAR*D study, MDD-AS (HAM-D-AFS score $\geq 7$ ) was associated with delayed and less likely remission compared with MDD without anxiety symptoms. Similarly, in Phase 2, MDD-AS was associated with poorer outcomes in both the switching (sustained-release bupropion/sertraline/venlafaxine ER), or augmentation (sustained-release bupropion/buspirone) options. ${ }^{120}$ Consistently, a pooled analysis of 13 double-blinded, randomized, controlled trials (including SSRIs and SNRIs) found that patients with MDD-AS (HAM-D-AFS score $\geq 7$ ) had lower remission rates than those without anxiety symptoms. ${ }^{121}$ In the GENDEP study, MDD-AS, based on HAM-D-AFS score, was not a predictor of outcome in the whole sample of participants; however, in secondary analyses restricted to randomly allocated participants, MDD-AS was associated with overall poorer outcomes. ${ }^{102}$ Multiple re-analyses of data from the iSPOT-D study ${ }^{32,116,122}$ found that greater anxiety symptoms at baseline, as measured with the anxiety subscale of the Depression, Anxiety and Stress Scale (DASS-42), were associated with lower response/remission rates across all medications, independent of depression severity and after adjustment for multiple covariates. The factor "somatic anxiety" appeared to 
be particularly related to worse outcomes. Conversely, no relationships between anxiety symptoms and outcome were found using the HAM-D-AFS scores. ${ }^{32,99,116,122}$ Finally, a recent longitudinal cohort study, the Netherlands Study of Depression and Anxiety, revealed that the DSM- 5 anxious distress specifier significantly predicted poorer outcomes with adequate antidepressant treatments in MDD, including SSRs, TCAs, and other antidepressants. Poorer outcomes were represented by higher depression severity (at 1-year and 2-year follow-up), lower remission rates (at 2-year follow-up), and greater burden of antidepressant side effects during treatment. Conversely, comorbidity with AnxDs did not predict treatment outcome. ${ }^{118,123}$ Across all the studies described above, no evidence of preferential response of MDD-AS to a specific antidepressant class or compound was found.

In conclusion, studies considering the dimensional definitions of anxiety in MDD point to associations between MDDAS and poorer responses to antidepressants, with a greater burden of side effects, suggesting careful monitoring of patients with these features during the course of antidepressant treatment. No indications are available for possible antidepressant-specific selection based on the presence of significant anxiety symptoms during an MDE.

\section{CONCLUSION AND FUTURE DIRECTIONS}

In the framework of personalized medicine in psychiatry, our review aimed to evaluate if some easily assessable sociodemographic and clinical variables may help clinicians to optimize selection of the most effective possible antidepressant treatment for each patient with MDD, at the early stage of the disorder. Unfortunately, gender, age, race/ethnicity, and SES, as well as BMI, severity of depressive symptoms, and symptom profiles (i.e., presence of anhedonia; MDD with melancholic features/atypical features/anxiety symptoms), when considered independently as separate variables, failed to provide value to predict reliable differences in benefits of alternative antidepressants for each patient. Several variables were associated with poorer outcomes to the whole group of antidepressants, such as low SES, genetic African ancestry, overweight/obesity, very severe pretreatment depressive symptoms, depression with melancholic symptoms, or with significant anxiety symptoms. However, only sparse and preliminary associations were found between some clinical variables (i.e., BMI, anhedonia, and MDD with melancholic/ atypical features) and possible benefits with some specific antidepressants, not sufficient to be considered as guidelines in choosing antidepressant treatment.

In conclusion, in clinical practice, the assessment of the sociodemographic and clinical variables considered in our review can be valuable for early identification of depressed individuals at high risk for poorer responses to antidepressants, who may require a particular setting and a higher intensity of care and monitoring. However, so far, there is not enough data on which to ground any reliable selection of specific antidepressant class or compound.

As discussed throughout the paper, the lack of reliable conclusions may be partly explained by limitations that this body of studies suffered from, including heterogeneity in design, duration, setting, features of the sample, statistical power, criteria and/or tools used to assess clinical symptoms or profiles, and the limited number of comparative studies testing the differential efficacy of specific antidepressants. In addition, it should be noted that our review focused only on a limited set of variables, while other individual/clinical factors, relatively easy to assess in clinical practice, may play a role, such as childhood maltreatment/adversities, ${ }^{124-126}$ inflammatory markers, ${ }^{127,128}$ other symptom profiles or individual features. Among the latter, alexithymia, reflecting difficulty with identifying and expressing emotions, seems to be associated with higher severity of depression, increased suicide risk, and pro-inflammatory imbalance; ${ }^{129-133}$ thus its potential contribute to selection of specific antidepressant class/compound may be worth investigating. Finally, we did not include combined pharmacotherapies, augmentation strategies, or non-pharmacological interventions. However, even if these limitations are overcome in the future, it appears unlikely that single variables, considered separately, may provide reliable indications for personalized treatment selection, as has been previously noted by others. Furthermore, it is claimed that refining the phenotypes of MDD beyond traditional psychiatric nosography, taking into account biomarkers, functional systems, neural circuits, and environmental factors, may be a crucial step in providing more reliable results both in pharmacological and genetic studies. ${ }^{134}$ Likewise, future use of biomarkers, such as neural markers in neuroimaging measures, may help clinicians distinguish depression in bipolar disorder from MDD. ${ }^{135}$ Indeed, mainly at the early stage of the disorder, this distinction, which is crucial for choosing appropriate treatments, is difficult when solely based on clinical information.

In this framework, the National Institute of Mental Health Research Domain Criteria ( $\mathrm{RDoC}$ ) initiative ${ }^{136}$ is ongoing, with the final aim of defining more reliable phenotypes for each depressed individual, based on which personalized therapies may be selected in the future. ${ }^{137,138}$

Consistent with this view, recent approaches have pointed to the development of composite prediction models that integrate multiple potential predictors through new technological acquisitions and computational resources, such as ma- 
chine learning and related techniques. ${ }^{139,140}$ Some promising attempts were performed, showing that this paradigm may offer relevant advances toward personalized treatments. A deep learning prediction approach, resulting from the integrated analysis of genetic (i.e., single nucleotide polymorphisms), sociodemographic, and clinical factors in a large sample of patients with MDD, seems to be suitable to distinguish responders from non-responders to SSRI treatment. ${ }^{141}$ A machine learning model recently developed using data from STAR*D (Phase 1) and CO-MED studies performed significantly-above-chance in predicting clinical remission to citalopram, escitalopram, and escitalopram-bupropion treatment, but not to venlafaxine-mirtazapine treatment, suggesting specificity of the model to identify patients who are likely to respond to a specific antidepressant. ${ }^{142}$ Similarly, a combination of demographic and clinical variables, obtained with a machine learning approach on data from the GENDEP study, provided a significant contribution in predicting response and remission in MDD during treatment with escitalopram, but not with nortriptyline, suggesting a potential for individualized prescription of this specific antidepressant. ${ }^{143}$ In a subsequent re-analysis of the data from the same sample, significant drugspecific predictions of remission for both escitalopram and nortriptyline were obtained, using statistical learning on a larger number of factors, including not only clinical variables but also common genetic variants. ${ }^{144}$ These encouraging results offer hope for the future, when predictions that are more reliable may guide personalized choices for each patient with MDD.

\section{Acknowledgments}

The authors would like to thank Enago (www.enago.com) for the English language review.

Giampaolo Perna, Alessandra Alciati, Silvia Daccò, Massimiliano Grassi, and Daniela Caldirola are scientific consultants for Medibio LTD. Giampaolo Perna has served as consultant for Lundbeck and Pfizer.

\section{Conflicts of Interest}

The authors have no potential conflicts of interest to disclose.

\section{Author Contributions}

All authors equally contributed to the selection and reviewing process of the scientific articles and the writing of the manuscript.

\section{ORCID iDs}

$\begin{array}{ll}\text { Giampaolo Perna } & \text { https://orcid.org/0000-0002-8166-0785 } \\ \text { Alessandra Alciati } & \text { https://orcid.org/0000-0002-8971-4696 } \\ \text { Silvia Daccò } & \text { https://orcid.org/0000-0002-7253-4808 } \\ \text { Massimiliano Grassi } & \text { https://orcid.org/0000-0002-7054-2644 } \\ \text { Daniela Caldirola } & \text { https://orcid.org/0000-0002-1461-9475 }\end{array}$

\section{REFERENCES}

1. Murray CJ, Atkinson C, Bhalla K, Birbeck G, Burstein R, Chou D, et al. The state of US health, 1990-2010: burden of diseases, injuries, and risk factors. JAMA 2013;310:591-608.
2. World Federation for Mental Health. Depression: A Global Crisis. Occoquan: World Federation for Mental Health; 2012.

3. Greenberg PE, Fournier AA, Sisitsky T, Pike CT, Kessler RC. The economic burden of adults with major depressive disorder in the United States (2005 and 2010). J Clin Psychiatry 2015;76:155-162.

4. Kennedy SH, Lam RW, McIntyre RS, Tourjman SV, Bhat V, Blier P, et al. Canadian Network for Mood and Anxiety Treatments (CANMAT) 2016 Clinical Guidelines for the Management of Adults with Major Depressive Disorder: Section 3. Pharmacological Treatments. Can J Psychiatry 2016;61:540-560.

5. Fornaro M, Anastasia A, Valchera A, Carano A, Orsolini L, Vellante F, et al. The FDA "Black Box" warning on antidepressant suicide risk in young adults: more harm than benefits? Front Psychiatry 2019;10: 294.

6. Carvalho AF, Cavalcante JL, Castelo MS, Lima MC. Augmentation strategies for treatment-resistant depression: a literature review. J Clin Pharm Ther 2007;32:415-428.

7. Gartlehner G, Hansen RA, Morgan LC, Thaler K, Lux L, Van Noord $\mathrm{M}$, et al. Comparative benefits and harms of second-generation antidepressants for treating major depressive disorder: an updated metaanalysis. Ann Intern Med 2011;155:772-785.

8. Trivedi MH, Greer TL, Grannemann BD, Chambliss HO, Jordan AN. Exercise as an augmentation strategy for treatment of major depression. J Psychiatr Pract 2006;12:205-213.

9. Lenox-Smith AJ, Jiang Q. Venlafaxine extended release versus citalopram in patients with depression unresponsive to a selective serotonin reuptake inhibitor. Int Clin Psychopharmacol 2008;23:113-119.

10. Rush AJ, Trivedi MH, Wisniewski SR, Nierenberg AA, Stewart JW, Warden D, et al. Acute and longer-term outcomes in depressed outpatients requiring one or several treatment steps: a STAR*D report. Am J Psychiatry 2006;163:1905-1917.

11. Blier P, Ward HE, Tremblay P, Laberge L, Hebert C, Bergeron R. Combination of antidepressant medications from treatment initiation for major depressive disorder: a double-blind randomized study. Am J Psychiatry 2010;167:281-288.

12. Rush AJ, Trivedi MH, Stewart JW, Nierenberg AA, Fava M, Kurian BT, et al. Combining medications to enhance depression outcomes (CO-MED): acute and long-term outcomes of a single-blind randomized study. Am J Psychiatry 2011;168:689-701.

13. Fried EI, Nesse RM. Depression is not a consistent syndrome: An investigation of unique symptom patterns in the STAR*D study. J Affect Disord 2015;172:96-102.

14. American Psychiatric Association. Diagnostic and Statistical Manual of Mental Disorders (5th Ed.). Arlington, VA: American Psychiatric Association; 2013.

15. Culpepper L, Muskin PR, Stahl SM. Major depressive disorder: understanding the significance of residual symptoms and balancing efficacy with tolerability. Am J Med 2015;128(9 Suppl):S1-S15.

16. Akechi T, Mantani A, Kurata K, Hirota S, Shimodera S, Yamada M, et al. Predicting relapse in major depression after successful initial pharmacological treatment. J Affect Disord 2019;250:108-113.

17. Fekadu A, Wooderson SC, Markopoulo K, Donaldson C, Papadopoulos A, Cleare AJ. What happens to patients with treatment-resistant depression? A systematic review of medium to long term outcome studies. J Affect Disord 2009;116:4-11.

18. Hamilton J, Grant M, Jensvold M. Sex and Treatment of Depression. In: M. Jensvold UH, Editor. Psychopharmacology and Women. Sex, Gender and Hormones. Washington, DC: American Psychiatric Association, 1996, p.241-260.

19. Kornstein SG, Schatzberg AF, Thase ME, Yonkers KA, McCullough JP, Keitner GI, et al. Gender differences in treatment response to sertraline versus imipramine in chronic depression. Am J Psychiatry 2000;157:1445-1452.

20. Khan A, Brodhead AE, Schwartz KA, Kolts RL, Brown WA. Sex differences in antidepressant response in recent antidepressant clinical 
trials. J Clin Psychopharmacol 2005;25:318-324.

21. Serretti A, Gibiino S, Drago A. Specificity profile of paroxetine in major depressive disorder: meta-regression of double-blind, randomized clinical trials. J Affect Disord 2011;132:14-25.

22. Gibiino S, Marsano A, Serretti A. Specificity profile of venlafaxine and sertraline in major depression: metaregression of double-blind, randomized clinical trials. Int J Neuropsychopharmacol 2014;17:1-8.

23. Berlanga C, Flores-Ramos M. Different gender response to serotonergic and noradrenergic antidepressants. A comparative study of the efficacy of citalopram and reboxetine. J Affect Disord 2006;95:119123.

24. Thase ME, Entsuah R, Cantillon M, Kornstein SG. Relative antidepressant efficacy of venlafaxine and SSRIs: sex-age interactions. J Womens Health (Larchmt) 2005;14:609-616.

25. Trivedi MH, Rush AJ, Wisniewski SR, Nierenberg AA, Warden D, Ritz L, et al. Evaluation of outcomes with citalopram for depression using measurement-based care in STAR*D: implications for clinical practice. Am J Psychiatry 2006;163:28-40.

26. Quitkin FM, Stewart JW, McGrath PJ, Taylor BP, Tisminetzky MS, Petkova E, et al. Are there differences between women's and men's antidepressant responses? Am J Psychiatry 2002;159:1848-1854.

27. Bagby RM, Ryder AG, Cristi C. Psychosocial and clinical predictors of response to pharmacotherapy for depression. J Psychiatry Neurosci 2002;27:250-257.

28. Lewis-Hall FC, Wilson MG, Tepner RG, Koke SC. Fluoxetine vs. tricyclic antidepressants in women with major depressive disorder. J Womens Health 1997;6:337-343.

29. Kornstein SG, Pedersen RD, Holland PJ, Nemeroff CB, Rothschild AJ, Thase ME, et al. Influence of sex and menopausal status on response, remission, and recurrence in patients with recurrent major depressive disorder treated with venlafaxine extended release or fluoxetine: analysis of data from the PREVENT study. J Clin Psychiatry 2014;75:62-68.

30. Wohlfarth T, Storosum JG, Elferink AJ, van Zwieten BJ, Fouwels A van den Brink W. Response to tricyclic antidepressants: independent of gender? Am J Psychiatry 2004;161:370-372.

31. Cuijpers P, Weitz E, Twisk J, Kuehner C, Cristea I, David D, et al. Gender as predictor and moderator of outcome in cognitive behavior therapy and pharmacotherapy for adult depression: an "individual patient data" meta-analysis. Depress Anxiety 2014;31:941-951.

32. Saveanu R, Etkin A, Duchemin AM, Goldstein-Piekarski A, Gyurak A, Debattista C, et al. The international Study to Predict Optimized Treatment in Depression (iSPOT-D): outcomes from the acute phase of antidepressant treatment. J Psychiatr Res 2015;61:1-12.

33. Kessler RC, van Loo HM, Wardenaar KJ, Bossarte RM, Brenner LA, Ebert DD, et al. Using patient self-reports to study heterogeneity of treatment effects in major depressive disorder. Epidemiol Psychiatr Sci 2017;26:22-36.

34. Noma H, Furukawa TA, Maruo K, Imai H, Shinohara K, Tanaka S, et al. Exploratory analyses of effect modifiers in the antidepressant treatment of major depression: individual-participant data meta-analysis of 2803 participants in seven placebo-controlled randomized trials. J Affect Disord 2019;250:419-424

35. Webb CA, Trivedi MH, Cohen ZD, Dillon DG, Fournier JC, Goer F, et al. Personalized prediction of antidepressant v. placebo response: evidence from the EMBARC study. Psychol Med 2019;49:1118-1127.

36. Marcos LR, Cancro R. Pharmacotherapy of Hispanic depressed patients: clinical observations. Am J Psychother 1982;36:505-512.

37. Varner RV, Ruiz P, Small DR. Black and white patients response to antidepressant treatment for major depression. Psychiatr Q 1998;69: $117-125$.

38. Miranda J, Schoenbaum M, Sherbourne C, Duan N, Wells K. Effects of primary care depression treatment on minority patients' clinical status and employment. Arch Gen Psychiatry 2004;61:827-834.

39. Wagner GJ, Maguen S, Rabkin JG. Ethnic differences in response to fluoxetine in a controlled trial with depressed HIV-positive patients. Psychiatr Serv 1998;49:239-240.

40. Bailey RK, Mallinckrodt CH, Wohlreich MM, Watkin JG, Plewes JM. Duloxetine in the treatment of major depressive disorder: comparisons of safety and efficacy. J Natl Med Assoc 2006;98:437-447.

41. Roy-Byrne PP, Perera P, Pitts CD, Christi JA. Paroxetine response and tolerability among ethnic minority patients with mood or anxiety disorders: a pooled analysis. J Clin Psychiatry 2005;66:1228-1233.

42. Lesser IM, Castro DB, Gaynes BN, Gonzalez J, Rush AJ, Alpert JE, et al. Ethnicity/race and outcome in the treatment of depression: results from STAR*D. Med Care 2007;45:1043-1051.

43. Lesser IM, Myers HF, Lin KM, Bingham Mira C, Joseph NT, Olmos NT, et al. Ethnic differences in antidepressant response: a prospective multi-site clinical trial. Depress Anxiety 2010;27:56-62.

44. Lesser IM, Zisook S, Gaynes BN, Wisniewski SR, Luther JF, Fava M, et al. Effects of race and ethnicity on depression treatment outcomes: the CO-MED trial. Psychiatr Serv 2011;62:1167-1179.

45. Murphy E, Hou L, Maher BS, Woldehawariat G, Kassem L, Akula N, et al. Race, genetic ancestry and response to antidepressant treatment for major depression. Neuropsychopharmacology 2013;38:2598-2606.

46. Murphy EJ, Kassem L, Chemerinski A, Rush AJ, Laje G, McMahon FJ. Retention and attrition among African Americans in the STAR ${ }^{*} \mathrm{D}$ study: what causes research volunteers to stay or stray? Depress Anxiety 2013;30:1137-1144.

47. Freeman A, Tyrovolas S, Koyanagi A, Chatterji S, Leonardi M, Ayuso-Mateos JL, et al. The role of socio-economic status in depression: results from the COURAGE (aging survey in Europe). BMC Public Health 2016;16:1098.

48. Lorant V, Croux C, Weich S, Deliege D, Mackenbach J, Ansseau M. Depression and socio-economic risk factors: 7-year longitudinal population study. Br J Psychiatry 2007;190:293-298.

49. Jakubovski E, Bloch MH. Prognostic subgroups for citalopram response in the STAR*D trial. J Clin Psychiatry 2014;75:738-747.

50. Perlman K, Benrimoh D, Israel S, Rollins C, Brown E, Tunteng JF, et al. A systematic meta-review of predictors of antidepressant treatment outcome in major depressive disorder. J Affect Disord 2019; 243:503-515.

51. Van HL, Schoevers RA, Dekker J. Predicting the outcome of antidepressants and psychotherapy for depression: a qualitative, systematic review. Harv Rev Psychiatry 2008;16:225-234.

52. Mandelli L, Serretti A, Souery D, Mendlewicz J, Kasper S, Montgomery $\mathrm{S}$, et al. High occupational level is associated with poor response to treatment of depression. Eur Neuropsychopharmacol 2016;26: 1320-1326.

53. Mandelli L, Serretti A, Porcelli S, Souery D, Mendlewicz J, Kasper S, et al. Opinion paper: poor response to treatment of depression in people in high occupational levels. Psychol Med 2019;49:49-54.

54. de Wit L, Luppino F, van Straten A, Penninx B, Zitman F, Cuijpers P. Depression and obesity: a meta-analysis of community-based studies. Psychiatry Res 2010;178:230-235.

55. Opel N, Redlich R, Grotegerd D, Dohm K, Heindel W, Kugel H, et al. Obesity and major depression: Body-mass index (BMI) is associated with a severe course of disease and specific neurostructural alterations. Psychoneuroendocrinology 2015;51:219-226.

56. Oskooilar N, Wilcox CS, Tong ML, Grosz DE. Body mass index and response to antidepressants in depressed research subjects. J Clin Psychiatry 2009;70:1609-1610.

57. Khan A, Schwartz KA, Kolts RL, Brown WA. BMI, sex, and antidepressant response. J Affect Disord 2007;99:101-106.

58. Lin CH, Chen CC, Wong J, McIntyre RS. Both body weight and BMI predicts improvement in symptom and functioning for patients with major depressive disorder. J Affect Disord 2014;161:123-126.

59. Papakostas GI, Petersen T, Iosifescu DV, Burns AM, Nierenberg AA, Alpert JE, et al. Obesity among outpatients with major depressive disorder. Int J Neuropsychopharmacol 2005;8:59-63. 
60. Uher R, Mors O, Hauser J, Rietschel M, Maier W, Kozel D, et al. Body weight as a predictor of antidepressant efficacy in the GENDEP project. J Affect Disord 2009;118:147-154.

61. Jantaratnotai N, Mosikanon K, Lee Y, McIntyre RS. The interface of depression and obesity. Obes Res Clin Pract 2017;11:1-10.

62. Woo YS, Seo HJ, McIntyre RS, Bahk WM. Obesity and its potential effects on antidepressant treatment outcomes in patients with depressive disorders: a literature review. Int J Mol Sci 2016;17. pii:E80.

63. Green E, Goldstein-Piekarskia AN, Schatzberg AF, Rush JA, Jun M, Williams L. Personalizing antidepressant choice by sex, body mass index, and symptom profile: An iSPOT-D report. Pers Med Psychiatry 2017;1-2:65-73.

64. Jha MK, Wakhlu S, Dronamraju N, Minhajuddin A, Greer TL, Trivedi $\mathrm{MH}$. Validating pre-treatment body mass index as moderator of antidepressant treatment outcomes: Findings from CO-MED trial. J Affect Disord 2018;234:34-37.

65. Khan A, Leventhal RM, Khan SR, Brown WA. Severity of depression and response to antidepressants and placebo: an analysis of the Food and Drug Administration database. J Clin Psychopharmacol 2002;22: 40-45.

66. Fournier JC, DeRubeis RJ, Hollon SD, Dimidjian S, Amsterdam JD, Shelton RC, et al. Antidepressant drug effects and depression severity: a patient-level meta-analysis. JAMA 2010;303:47-53.

67. Friedman ES, Davis LL, Zisook S, Wisniewski SR, Trivedi MH, Fava $\mathrm{M}$, et al. Baseline depression severity as a predictor of single and combination antidepressant treatment outcome: results from the COMED trial. Eur Neuropsychopharmacol 2012;22:183-199.

68. Bartova L, Dold M, Kautzky A, Fabbri C, Spies M, Serretti A, et al. Results of the European Group for the Study of Resistant Depression (GSRD)-basis for further research and clinical practice. World J Biol Psychiatry 2019;20:427-448.

69. National Institute for Health and Care Excellence (NICE). Depression in Adults: Recognition and Management NICE. https://www.nice. org.uk/guidance/cg90. Clinical Guideline [CG90]; 2009.

70. American Psychiatric Association. Diagnostic and Statistical Manual of Mental Disorders (4th Ed., Text Revision). Washington, DC: American Psychiatric Pub; 2000.

71. Pizzagalli DA. Depression, stress, and anhedonia: toward a synthesis and integrated model. Annu Rev Clin Psychol 2014;10:393-423.

72. Berridge KC, Robinson TE. Parsing reward. Trends Neurosci 2003; 26:507-513.

73. Clery-Melin ML, Jollant F, Gorwood P. Reward systems and cognitions in Major Depressive Disorder. CNS Spectr 2019;24:64-77.

74. Lamontagne SJ, Melendez SI, Olmstead MC. Investigating dopamine and glucocorticoid systems as underlying mechanisms of anhedonia. Psychopharmacology (Berl) 2018;235:3103-3113.

75. Treadway MT, Zald DH. Reconsidering anhedonia in depression: lessons from translational neuroscience. Neurosci Biobehav Rev 2011; 35:537-555.

76. Haroon E, Chen X, Li Z, Patel T, Woolwine BJ, Hu XP, et al. Increased inflammation and brain glutamate define a subtype of depression with decreased regional homogeneity, impaired network integrity, and anhedonia. Transl Psychiatry 2018;8:189.

77. Davidson KW, Burg MM, Kronish IM, Shimbo D, Dettenborn L, Mehran $\mathrm{R}$, et al. Association of anhedonia with recurrent major adverse cardiac events and mortality 1 year after acute coronary syndrome. Arch Gen Psychiatry 2010;67:480-488.

78. Fawcett J, Scheftner WA, Fogg L, Clark DC, Young MA, Hedeker D, et al. Time-related predictors of suicide in major affective disorder. Am J Psychiatry 1990;147:1189-1194.

79. Spijker J, Bijl RV, de Graaf R, Nolen WA. Determinants of poor 1-year outcome of DSM-III-R major depression in the general population: results of the Netherlands Mental Health Survey and Incidence Study (NEMESIS). Acta Psychiatr Scand 2001;103:122-130.

80. Vrieze E, Pizzagalli DA, Demyttenaere K, Hompes T, Sienaert P, de
Boer P, et al. Reduced reward learning predicts outcome in major depressive disorder. Biol Psychiatry 2013;73:639-645.

81. Uher R, Perlis RH, Henigsberg N, Zobel A, Rietschel M, Mors O, et al. Depression symptom dimensions as predictors of antidepressant treatment outcome: replicable evidence for interest-activity symptoms. Psychol Med 2012;42:967-980.

82. Dunlop BW, Nemeroff CB. The role of dopamine in the pathophysiology of depression. Arch Gen Psychiatry 2007;64:327-337.

83. Nutt D, Demyttenaere K, Janka Z, Aarre T, Bourin M, Canonico PL, et al. The other face of depression, reduced positive affect: the role of catecholamines in causation and cure. J Psychopharmacol 2007;21: 461-471.

84. McCabe C, Mishor Z, Cowen PJ, Harmer CJ. Diminished neural processing of aversive and rewarding stimuli during selective serotonin reuptake inhibitor treatment. Biol Psychiatry 2010;67:439-445.

85. Goodwin GM, Price J, De Bodinat C, Laredo J. Emotional blunting with antidepressant treatments: A survey among depressed patients. J Affect Disord 2017;221:31-35.

86. Blier P, El Mansari M. Serotonin and beyond: therapeutics for major depression. Philos Trans R Soc Lond B Biol Sci 2013;368:20120536.

87. Blier P, Briley M. The noradrenergic symptom cluster: clinical expression and neuropharmacology. Neuropsychiatr Dis Treat 2011;7 (Suppl 1):15-20.

88. Cao B, Zhu J, Zuckerman H, Rosenblat JD, Brietzke E, Pan Z, et al. Pharmacological interventions targeting anhedonia in patients with major depressive disorder: A systematic review. Prog Neuropsychopharmacol Biol Psychiatry 2019;92:109-117.

89. De Berardis D, Marini S, Fornaro M, Srinivasan V, Iasevoli F, Tomasetti $\mathrm{C}$, et al. The melatonergic system in mood and anxiety disorders and the role of agomelatine: implications for clinical practice. Int J Mol Sci 2013;14:12458-12483.

90. Martinotti G, Pettorruso M, De Berardis D, Varasano PA, Lucidi Pressanti G, De Remigis V, et al. Agomelatine increases BDNF serum levels in depressed patients in correlation with the improvement of depressive symptoms. Int J Neuropsychopharmacol 2016;19. pii:pyw003.

91. Martinotti G, Sepede G, Gambi F, Di Iorio G, De Berardis D, Di Nicola $\mathrm{M}$, et al. Agomelatine versus venlafaxine XR in the treatment of anhedonia in major depressive disorder: a pilot study. J Clin Psychopharmacol 2012;32:487-491.

92. Corruble E, de Bodinat C, Belaidi C, Goodwin GM; agomelatine study group. Efficacy of agomelatine and escitalopram on depression, subjective sleep and emotional experiences in patients with major depressive disorder: a 24 -wk randomized, controlled, double-blind trial. Int J Neuropsychopharmacol 2013;16:2219-2234.

93. Light SN, Heller AS, Johnstone T, Kolden GG, Peterson MJ, Kalin NH, et al. Reduced right ventrolateral prefrontal cortex activity while inhibiting positive affect is associated with improvement in hedonic capacity after 8 weeks of antidepressant treatment in major depressive disorder. Biol Psychiatry 2011;70:962-968.

94. Jouvent R, Le Houezec J, Payan C, Mikkelsen H, Fermanian J, Millet $\mathrm{V}$, et al. Dimensional assessment of onset of action of antidepressants: a comparative study of moclobemide vs. clomipramine in depressed patients with blunted affect and psychomotor retardation. Psychiatry Res 1998;79:267-275.

95. Tomarken AJ, Dichter GS, Freid C, Addington S, Shelton RC. Assessing the effects of bupropion SR on mood dimensions of depression. J Affect Disord 2004;78:235-241.

96. Lally N, Nugent AC, Luckenbaugh DA, Niciu MJ, Roiser JP Zarate CA, Jr. Neural correlates of change in major depressive disorder anhedonia following open-label ketamine. J Psychopharmacol 2015;29: 596-607.

97. McGrath PJ, Khan AY, Trivedi MH, Stewart JW, Morris DW, Wisniewski SR, et al. Response to a selective serotonin reuptake inhibitor (citalopram) in major depressive disorder with melancholic features: a STAR*D report. J Clin Psychiatry 2008;69:1847-1855. 
98. Day CV, John Rush A, Harris AW, Boyce PM, Rekshan W, Etkin A, et al. Impairment and distress patterns distinguishing the melancholic depression subtype: an iSPOT-D report. J Affect Disord 2015;174:493502.

99. Arnow BA, Blasey C, Williams LM, Palmer DM, Rekshan W, Schatzberg AF, et al. Depression subtypes in predicting antidepressant response: a report from the iSPOT-D trial. Am J Psychiatry 2015;172: 743-750.

100. Bobo WV, Chen H, Trivedi MH, Stewart JW, Nierenberg AA, Fava $\mathrm{M}$, et al. Randomized comparison of selective serotonin reuptake inhibitor (escitalopram) monotherapy and antidepressant combination pharmacotherapy for major depressive disorder with melancholic features: a CO-MED report. J Affect Disord 2011;133:467-476.

101. Gili M, Roca M, Armengol S, Asensio D, Garcia-Campayo J, Parker G. Clinical patterns and treatment outcome in patients with melancholic, atypical and non-melancholic depressions. PLoS One 2012;7: e48200.

102. Uher R, Dernovsek MZ, Mors O, Hauser J, Souery D, Zobel A, et al. Melancholic, atypical and anxious depression subtypes and outcome of treatment with escitalopram and nortriptyline. J Affect Disord 2011; 132:112-120.

103. Yang SJ, Stewart R, Kang HJ, Kim SY, Bae KY, Kim JM, et al. Response to antidepressants in major depressive disorder with melancholic features: the CRESCEND study. J Affect Disord 2013;144:42-50.

104. Valerio MP, Szmulewicz AG, Martino DJ. A quantitative review on outcome-to-antidepressants in melancholic unipolar depression. Psychiatry Res 2018;265:100-110.

105. Buhler J, Seemuller F, Lage D. The predictive power of subgroups: an empirical approach to identify depressive symptom patterns that predict response to treatment. J Affect Disord 2014;163:81-87.

106. Stewart JW, McGrath PJ, Fava M, Wisniewski SR, Zisook S, Cook I, et al. Do atypical features affect outcome in depressed outpatients treated with citalopram? Int J Neuropsychopharmacol 2010;13:15-30.

107. Stewart JW, Garfinkel R, Nunes EV, Donovan S, Klein DF. Atypical features and treatment response in the National Institute of Mental Health Treatment of Depression Collaborative Research Program. J Clin Psychopharmacol 1998;18:429-434.

108. Quitkin FM, Stewart JW, McGrath PJ, Tricamo E, Rabkin JG, Ocepek-Welikson K, et al. Columbia atypical depression. A subgroup of depressives with better response to MAOI than to tricyclic antidepressants or placebo. Br J Psychiatry Suppl 1993;(21):30-34.

109. Lonnqvist J, Sihvo S, Syvalahti E, Kiviruusu O. Moclobemide and fluoxetine in atypical depression: a double-blind trial. J Affect Disord 1994;32:169-177.

110. Sogaard J, Lane R, Latimer P, Behnke K, Christiansen PE, Nielsen B, et al. A 12-week study comparing moclobemide and sertraline in the treatment of outpatients with atypical depression. J Psychopharmacol 1999;13:406-414.

111. Henkel V, Mergl R, Allgaier AK, Kohnen R, Moller HJ, Hegerl U. Treatment of depression with atypical features: a meta-analytic approach. Psychiatry Res 2006;141:89-101.

112. Bech P, Stage KB, Larsen JK, Vestergaard P, Gram LF; Danish University Antidepressant Group (DUAG). The predictive validity of atypical neurovegetative depressive symptoms identified by the first principal component in the DUAG trial of moclobemide versus clomipramine. J Affect Disord 2012;140:253-259.

113. Lojko D, Rybakowski JK. Atypical depression: current perspectives. Neuropsychiatr Dis Treat 2017;13:2447-2456.

114. Lojko D, Buzuk G, Owecki M, Ruchala M, Rybakowski JK. Atypical features in depression: Association with obesity and bipolar disorder. J Affect Disord 2015;185:76-80.

115. Milaneschi Y, Lamers F, Bot M, Drent ML, Penninx BW. Leptin dysregulation is specifically associated with major depression with atypical features: evidence for a mechanism connecting obesity and depression. Biol Psychiatry 2017;81:807-814.
116. Braund TA, Palmer DM, Williams LM, Harris AW. Characterising anxiety in major depressive disorder and its use in predicting antidepressant treatment outcome: an iSPOT-D report. Aust N Z J Psychiatry 2019;53:782-793.

117. Gaspersz R, Nawijn L, Lamers F, Penninx B. Patients with anxious depression: overview of prevalence, pathophysiology and impact on course and treatment outcome. Curr Opin Psychiatry 2018;31:17-25.

118. Gaspersz R, Lamers F, Kent JM, Beekman AT, Smit JH, van Hemert $\mathrm{AM}$, et al. Longitudinal predictive validity of the DSM-5 anxious distress specifier for clinical outcomes in a large cohort of patients with major depressive disorder. J Clin Psychiatry 2017;78:207-213.

119. Ionescu DF, Niciu MJ, Richards EM, Zarate CA Jr. Pharmacologic treatment of dimensional anxious depression: a review. Prim Care Companion CNS Disord 2014;16.

120. Fava M, Rush AJ, Alpert JE, Balasubramani GK, Wisniewski SR, Carmin CN, et al. Difference in treatment outcome in outpatients with anxious versus nonanxious depression: a STAR*D report. Am J Psychiatry 2008;165:342-351.

121. Papakostas GI, Larsen K. Testing anxious depression as a predictor and moderator of symptom improvement in major depressive disorder during treatment with escitalopram. Eur Arch Psychiatry Clin Neurosci 2011;261:147-156.

122. Braund TA, Palmer DM, Williams LM, Harris AWF. Dimensions of anxiety in Major depressive disorder and their use in predicting antidepressant treatment outcome: an iSPOT-D report. Psychol Med 2019:1-11. [Epub ahead of print]

123. Gaspersz R, Lamers F, Kent JM, Beekman ATF, Smit JH, van Hemert $\mathrm{AM}$, et al. Anxious distress predicts subsequent treatment outcome and side effects in depressed patients starting antidepressant treatment. J Psychiatr Res 2017;84:41-48.

124. Liu RT. Childhood adversities and depression in adulthood: current findings and future directions. Clin Psychol (New York) 2017;24:140153.

125. Nemeroff CB, Heim CM, Thase ME, Klein DN, Rush AJ, Schatzberg $\mathrm{AF}$, et al. Differential responses to psychotherapy versus pharmacotherapy in patients with chronic forms of major depression and childhood trauma. Proc Natl Acad Sci U S A 2003;100:14293-14296.

126. Williams LM, Debattista C, Duchemin AM, Schatzberg AF, Nemeroff CB. Childhood trauma predicts antidepressant response in adults with major depression: data from the randomized international study to predict optimized treatment for depression. Transl Psychiatry 2016; 6:e799.

127. Himmerich H, Patsalos O, Lichtblau N, Ibrahim MAA, Dalton B. Cytokine research in depression: principles, challenges, and open questions. Front Psychiatry 2019;10:30.

128. Strawbridge R, Arnone D, Danese A, Papadopoulos A, Herane Vives A, Cleare AJ. Inflammation and clinical response to treatment in depression: A meta-analysis. Eur Neuropsychopharmacol 2015;25:15321543.

129. De Berardis D, Conti C, Iasevoli F, Valchera A, Fornaro M, Cavuto M, et al. Alexithymia and its relationships with acute phase proteins and cytokine release: an updated review. J Biol Regul Homeost Agents 2014;28:795-799.

130. De Berardis D, Fornaro M, Orsolini L, Valchera A, Carano A, Vellante $\mathrm{F}$, et al. Alexithymia and suicide risk in psychiatric disorders: a minireview. Front Psychiatry 2017;8:148.

131. De Berardis D, Fornaro M, Valchera A, Rapini G, Di Natale S, De Lauretis I, et al. Alexithymia, resilience, somatic sensations and their relationships with suicide ideation in drug naive patients with firstepisode major depression: An exploratory study in the "real world" everyday clinical practice. Early Interv Psychiatry 2019 [Epub ahead of print].

132. De Berardis D, Olivieri L, Rapini G, Di Natale S, Serroni N, Fornaro $\mathrm{M}$, et al. Alexithymia, suicide ideation and homocysteine levels in drug naive patients with major depression: a study in the "Real World" 
clinical practice. Clin Psychopharmacol Neurosci 2019;17: 318-322.

133. Hemming L, Taylor P, Haddock G, Shaw J, Pratt D. A systematic review and meta-analysis of the association between alexithymia and suicide ideation and behaviour. J Affect Disord 2019;254:34-48.

134. Kaufman J. Unraveling the genetics of major depression and stressrelated psychiatric disorders: is it time for a paradigm shift? Biol Psychiatry 2018;84:82-84.

135. Han KM, De Berardis D, Fornaro M, Kim YK. Differentiating between bipolar and unipolar depression in functional and structural MRI studies. Prog Neuropsychopharmacol Biol Psychiatry 2019;91:20-27.

136. National Institute of Mental Health. The National Institute of Mental Health Strategic Plan. Bethesda: National Institute of Mental Health; 2008.

137. Dean J, Keshavan M. The neurobiology of depression: An integrated view. Asian J Psychiatr 2017;27:101-111.

138. Sanislow CA, Ferrante M, Pacheco J, Rudorfer MV, Morris SE. Advancing translational research using NIMH research domain criteria and computational methods. Neuron 2019;101:779-782.

139. Kessler RC, Bossarte RM, Luedtke A, Zaslavsky AM, Zubizarreta JR.
Machine learning methods for developing precision treatment rules with observational data. Behav Res Ther 2019;120:103412.

140. Perna G, Grassi M, Caldirola D, Nemeroff CB. The revolution of personalized psychiatry: will technology make it happen sooner? Psychol Med 2018;48:705-713.

141. Lin E, Kuo PH, Liu YL, Yu YW, Yang AC, Tsai SJ. A deep learning approach for predicting antidepressant response in major depression using clinical and genetic biomarkers. Front Psychiatry 2018;9:290.

142. Chekroud AM, Zotti RJ, Shehzad Z, Gueorguieva R, Johnson MK, Trivedi MH, et al. Cross-trial prediction of treatment outcome in depression: a machine learning approach. Lancet Psychiatry 2016;3:243250.

143. Iniesta R, Malki K, Maier W, Rietschel M, Mors O, Hauser J, et al. Combining clinical variables to optimize prediction of antidepressant treatment outcomes. J Psychiatr Res 2016;78:94-102.

144. Iniesta R, Hodgson K, Stahl D, Malki K, Maier W, Rietschel M, et al. Antidepressant drug-specific prediction of depression treatment outcomes from genetic and clinical variables. Sci Rep 2018;8:5530. 